\title{
Genetic management strategies for controlling infectious diseases in livestock populations
}

\author{
Stephen C. Bishop*, Katrin M. MaCKenZIE** \\ Roslin Institute (Edinburgh), Roslin, Midlothian, EH25 9PS, UK
}

(Accepted 4 February 2003)

\begin{abstract}
This paper considers the use of disease resistance genes to control the transmission of infection through an animal population. Transmission is summarised by $\mathrm{R}_{0}$, the basic reproductive ratio of a pathogen. If $\mathrm{R}_{0}>1.0$ a major epidemic can occur, thus a disease control strategy should aim to reduce $\mathrm{R}_{0}$ below $1.0, e$.g. by mixing resistant with susceptible wild-type animals. Suppose there is a resistance allele, such that transmission of infection through a population homozygous for this allele will be $\mathrm{R}_{02}<\mathrm{R}_{01}$, where $\mathrm{R}_{01}$ describes transmission in the wildtype population. For an otherwise homogeneous population comprising animals of these two groups, $\mathbf{R}_{0}$ is the weighted average of the two sub-populations: $\mathbf{R}_{0}=R_{01} \rho+\mathbf{R}_{02}(1-\rho)$, where $\rho$ is the proportion of wildtype animals. If $R_{01}>1$ and $R_{02}<1$, the proportions of the two genotypes should be such that $\mathrm{R}_{0} \leq 1$, i.e. $\rho \leq\left(\mathrm{R}_{0}-\mathrm{R}_{02}\right) /\left(\mathrm{R}_{01}-\mathrm{R}_{02}\right)$. If $\mathrm{R}_{02}=0$, the proportion of resistant animals must be at least $1-1 / \mathrm{R}_{01}$. For an $n$ genotype model the requirement is still to have $\mathrm{R}_{0} \leq 1.0$. Probabilities of epidemics in genetically mixed populations conditional upon the presence of a single infected animal were derived. The probability of no epidemic is always $1 /\left(R_{0}+1\right)$. When $R_{0} \leq 1$ the probability of a minor epidemic, which dies out without intervention, is $\mathrm{R}_{0} /\left(\mathrm{R}_{0}+1\right)$. When $\mathrm{R}_{0}>1$ the probability of a minor and major epidemics are $1 /\left(\mathrm{R}_{0}+1\right)$ and $\left(\mathrm{R}_{0}-1\right) /\left(\mathrm{R}_{0}+1\right)$. Wherever possible a combination of genotypes should be used to minimise the invasion possibilities of pathogens that have mutated to overcome the effects of specific resistance alleles.
\end{abstract}

genetics / epidemiology / disease resistance / livestock / $\mathbf{R}_{\mathbf{0}}$

\section{INTRODUCTION}

Disease resistance is perhaps the major challenge facing animal geneticists. The long-term sustainability of many livestock sectors will depend upon having animals resistant to certain infectious diseases. In some cases, this might even become a requirement imposed upon livestock industries. Genetic variation in host resistance exists for many diseases [3] and much research effort is now

\footnotetext{
* Correspondence and reprints

E-mail: Stephen.Bishop@bbsrc.ac.uk

** Current address: BioSS, Scottish Crop Research Institute, Invergowrie, Dundee, UK
} 
directed towards finding disease resistance genes. An example of success is the identification of PrP gene controlling resistance of sheep to scrapie [4]. Many sheep industries in Western Europe are now required to select for scrapie resistance.

To design effective breeding strategies utilising resistance genes it is necessary to understand the impact these genes have on the transmission of infection, or disease severity, should animals become infected. Critical is the distinction between (i) resistance, the animal's ability to resist infection or moderate the parasite lifecycle and (ii) tolerance, the animal's ability to withstand the pathogenic effects of infection, i.e. disease. Improving tolerance will reduce the impact of infection, but it will have little direct effect upon the transmission of infection. Conversely, improving resistance will reduce the transmission of infection and therefore the severity of disease in the population. Deployment of resistance genes will only be worthwhile if it can be demonstrated that they will substantially reduce probability of epidemics, should the infectious agent be present, or the severity of an epidemic, should an epidemic take hold. Therefore, both genetic and epidemiological theory will be required to develop breeding strategies utilising resistance genes. For example, it has been shown that it is not necessary to make all animals genetically resistant to protect a population as a whole from epidemics [13], with the required proportion of resistant animals being a function of the infectiousness of the disease.

This paper provides a general framework for deriving strategies for utilising disease resistance genes in domestic livestock populations, particularly when the focus in on sporadic diseases caused by bacterial or viral infections with direct animal-to-animal transmission. Pathogen evolution in response to changing host genotype is often raised as a risk with genetic control strategies. Thus, this paper also considers scenarios under which pathogen evolution is more likely, and the impact that these scenarios have upon recommended strategies for utilising genes.

\section{METHODOLOGY}

\subsection{Background}

Transmission of infection through a population may be summarised by $\mathrm{R}_{0}$, the basic reproductive ratio. $\mathrm{R}_{0}$ may be defined as the number of secondary infections caused by the introduction of a single infected animal into an otherwise wholly susceptible population [1]. Deterministic expectations are such that if $\mathrm{R}_{0}$ is greater than 1.0 it is expected that an epidemic will occur upon the introduction of an infected animal in an otherwise healthy population, but if $\mathrm{R}_{0} \leq 1.0$ an epidemic is not expected [6]. Stochastic chance events ensure that for $\mathrm{R}_{0}$ values greater than 1.0 , there is a possibility that there will be no 
epidemic, or a minor epidemic which dies out without intervention (e.g. [14]). Likewise, for $\mathrm{R}_{0} \leq 1.0$, minor epidemics can arise.

By definition, a population comprised solely of genetically resistant animals results in the pathogen having an $\mathrm{R}_{0}$ of less than 1.0. This is irrespective of $\mathrm{R}_{0}$ in the population of genetically susceptible wild-type animals. Therefore, a rational aim of a disease control strategy is to reduce $R_{0}$ in the target population to below 1.0. This may be achieved by selecting animals for enhanced resistance or, in the case of discrete levels of resistance, by mixing genetically resistant animals with susceptible wild-type animals. This paper considers the case of resistance expressed in terms of categories, e.g. when one or a small number of genes confer resistance, and when resistance may either be complete (i.e. $\mathrm{R}_{0}<1$ ) or partial (i.e. $\left.\mathrm{R}_{0}>1\right)$.

\subsection{Required proportions of resistant animals}

Under assumptions of no spatial heterogeneity and equal contact amongst animals of different genotypes, i.e. a fully mixing genetically heterogeneous population, $\mathrm{R}_{0}$ for the population as a whole will be the weighted average of the $\mathrm{R}_{0}$ values within each subgroup [7]. Assume that for a given disease, if the host population comprises animals with a wild-type genotype, then transmission of infection is described by $R_{01}$. Now suppose that a resistance allele, $r$, is found, such that if the host population comprises animals homozygous for this allele transmission is altered to $R_{02}$, where $R_{02}<R_{01}$. Our objective is to use judicious selection of animals to construct a mixed population where $\mathbf{R}_{0}=\left(\mathbf{R}_{01} \rho_{1}+\mathbf{R}_{02}\left(1-\rho_{1}\right)\right)$, where $\rho_{1}$ is the proportion of wildtype animals. Hereafter this is called the two-genotype model.

The two-genotype model can be extended to the general case of $n$ genetic categories. These categories might represent animals that are combinations of, say, homozygous for the susceptibility allele, heterozygous and homozygous for the resistance allele, across several loci. Now there are $n$ levels of resistance, denoted by $\mathbf{R}_{01} \ldots \mathbf{R}_{0 \mathrm{n}}$, and a different proportion of animals $\left(\rho_{1}, \rho_{2}, \ldots \rho_{\mathrm{n}}\right)$ corresponding to each of these groups. Thus, $R_{0}=\left(R_{01} \rho_{1}+R_{02} \rho_{2}+\cdots\right.$ $\left.\cdots+\mathrm{R}_{0 \mathrm{n}} \rho_{\mathrm{n}}\right)$.

Consider the two-genotype model. There are three possible scenarios. Firstly, $\mathrm{R}_{01}<1$ in which case there is no disease problem. Secondly, $\mathrm{R}_{02}>1$ in which case the population will be susceptible to epidemics no matter what the genetic make up of the population. To minimise the probability or severity of epidemics, $\rho_{1}$ should be set to zero, reducing the epidemic risk by having all animals homozygous for the resistance allele. Thirdly, $\mathrm{R}_{01}>1$ and $\mathrm{R}_{02}<1$. Here, the requirement is to reduce $\mathrm{R}_{0}$ below 1.0, where $\mathrm{R}_{0}=\mathrm{R}_{01} \rho_{1}+\mathrm{R}_{02}\left(1-\rho_{1}\right)$. Thus:

$$
\rho_{1}=\left(\mathrm{R}_{0}-\mathrm{R}_{02}\right) /\left(\mathrm{R}_{01}-\mathrm{R}_{02}\right)
$$


where $\rho_{1}$ is the proportion of wild-type genotypes in the population, and $\mathrm{R}_{0}$ is some desired value between 0 and 1 . For the threshold case where $R_{0}$ is 1.0 and $\mathrm{R}_{02}$ is 0 (i.e. complete resistance) the proportion of resistant animals must be at least $1-1 / R_{01}$. This is conceptually equivalent to the proportion of animals requiring vaccination, under the assumption of a completely effective vaccine used in a fully mixed population [2], and it is also the result found by MacKenzie and Bishop [13].

For the $n$ genotype model, there is no single explicit solution to the number of animals required of each genotype. Rather, the aim is simply to combine the genotypic classes such that $\mathrm{R}_{0}<1$. For example, for a 3 genotype model, the requirement is simply to find values of $\rho_{1}$ and $\rho_{2}$ that solve the equation (obtained by rearrangement):

$$
\rho_{1}+\rho_{2}\left(\mathbf{R}_{02}-\mathrm{R}_{03}\right) /\left(\mathrm{R}_{01}-\mathrm{R}_{03}\right)=\left(\mathrm{R}_{0}-\mathrm{R}_{03}\right) /\left(\mathrm{R}_{01}-\mathrm{R}_{03}\right)
$$

Desired solutions to the $n$ genotype model will usually combine as many of the genotypes as feasible, unless some are highly susceptible. There will usually be sound biological, production and epidemiological reasons why this should be the case, including minimisation of pathogen co-evolution risks, as discussed below.

\subsection{Probability of epidemics}

The threshold $\mathrm{R}_{0}=1$ describes the value at which the probability of major epidemics becomes non-zero. However, above this threshold there is a probability of no epidemic occurring, and below the threshold there is a probability that a minor epidemic can occur. These probabilities, conditional upon the presence of a single infected animal, can be derived from epidemic theory appropriate to directly transmitted viral or bacterial infections. In such cases, transmission of infection can be described using an SIR model [2]. In this model, a population of $\mathrm{N}$ animals is made up of $\mathrm{S}$ susceptible, I infected and $\mathrm{R}$ recovered/removed animals. The model assumes no births, deaths or migration and full mixing between all animals in the population. Given a transmission rate, $\beta$, and a recovery rate $\gamma$, the rate of change of the numbers of animals in each category is described by the following equations:

$$
\begin{aligned}
\mathrm{dS} / \mathrm{dt} & =-\beta \mathrm{SI} \\
\mathrm{dI} / \mathrm{dt} & =\beta \mathrm{SI}-\gamma \mathrm{I} \\
\mathrm{dR} / \mathrm{dt} & =\gamma \mathrm{I} .
\end{aligned}
$$

The transmission rate, $\beta$, can be described specifically as $\mathrm{R}_{0} \gamma / \mathrm{N}$ [2]. Genetic heterogeneity can be incorporated by replacing $S$, I and R with $S_{i, t}, I_{i, t}$ and $R_{i, t}$ which represent the number of susceptible, infected or recovered animals 
of genotype $\mathrm{i}$ at time $\mathrm{t}$ such that $\mathrm{S}_{\mathrm{i}, \mathrm{t}}+\mathrm{I}_{\mathrm{i}, \mathrm{t}}+\mathrm{R}_{\mathrm{i}, \mathrm{t}}=\mathrm{N}_{\mathrm{i}, \mathrm{t}}$, under the assumption that the transmission rate is genotype dependent. With this assumption, and also assuming that the recovery rate is not genotype dependent, the genotype dependent transmission coefficients are denoted by $\beta_{\mathrm{i}}=\mathrm{R}_{0 \mathrm{i}} \gamma / \mathrm{N}_{\mathrm{i}}$ where $\mathrm{R}_{0 \mathrm{i}}$ is the basic reproductive ratio of the pathogen in a population with genotype $\mathrm{i}$.

These equations can be re-specified in a stochastic setting [15]. This is the first step in deriving epidemic probabilities, as it allows us to determine the probability of each type of event, i.e. the probability that a susceptible animal is infected and moves to the infected category or that an infected animal recovers. In a population where $\mathrm{R}_{0}<1.0$, a minor epidemic is defined as one where there is more than a single infected animal. The probability of a minor epidemic upon the introduction of a single infected animal can be estimated from the probability that the first event, after the introduction of an infected index case, is an infection rather than a recovery. Following the methodology of MacKenzie and Bishop [14] it is first necessary to construct the "rate" (K), of all possible events. In a situation where there is a single infected animal of genotype $\mathrm{i}$, the possible events, in a population with two genotypes, $i$ and $j$, are:

(i) infection of an animal with genotype $\mathrm{i}=\beta_{\mathrm{i}} \mathrm{S}_{\mathrm{i}} \mathrm{c}_{\mathrm{i}, \mathrm{i}}$

(ii) infection of an animal with genotype $\mathrm{j}=\beta_{\mathrm{j}} \mathrm{S}_{\mathrm{j}} \mathrm{c}_{\mathrm{j}, \mathrm{i}}$

(iii) recovery of the infected animal $=\gamma$

where $c_{j, i}$ is the contact rate between animals with genotype $i$ and animals with genotype $j$, which is simply the proportion of animals of type $j$ animals in the population. Therefore,

$$
\mathrm{K}=\beta_{\mathrm{i}} \mathrm{S}_{\mathrm{i}} \mathrm{c}_{\mathrm{i}, \mathrm{i}}+\beta_{\mathrm{j}} \mathrm{S}_{\mathrm{j}} \mathrm{c}_{\mathrm{j}, \mathrm{i}}+\gamma
$$

The probability of no epidemic is the probability that the first event is the recovery of the single infected animal, i.e. $\gamma / \mathrm{K}$. The probability that an epidemic occurs is $1-(\gamma / \mathrm{K})$. For $\mathbf{R}_{0}<1$ this is the probability of a minor epidemic, and for $\mathrm{R}_{0}>1$ it is the combined probability of a major or a minor epidemic.

The probability of no epidemic may be solved using the two-genotype model. Let $\mathrm{x}_{1}$ be the number of animals with the wild-genotype and $\mathrm{x}_{2}$ be the number of resistant animals in the population, then $\beta_{1}=\mathrm{R}_{01} \gamma / \mathrm{S}_{1}$ and $\beta_{2}=\mathrm{R}_{02} \gamma / \mathrm{S}_{2}$ are the transmission rates in groups of animals with the wild-genotype and resistance genotype respectively. With a single infected animal of the wildtype genotype, the contact rate between this animal and the susceptible animals of each genotype is proportional to the number of animals of each genotype, which is defined above (equation (1)) in terms of $R_{0}$ values. Therefore $c_{1,1}=$ $\left(\mathrm{R}_{0}-\mathrm{R}_{02}\right) /\left(\mathrm{R}_{01}-\mathrm{R}_{02}\right)$ and $c_{2,1}=\left(\mathrm{R}_{01}-\mathrm{R}_{0}\right) /\left(\mathrm{R}_{01}-\mathrm{R}_{02}\right)$, regardless of the $\mathrm{R}_{0}$ value. Substituting into equation (3) the probability of no epidemic is obtained 
as:

$$
\begin{aligned}
\frac{\gamma}{\mathrm{K}} & =\frac{\gamma}{\left[\left(\frac{\mathrm{R}_{0}-\mathrm{R}_{02}}{\mathrm{R}_{01}-\mathrm{R}_{02}}\right)\left(\frac{\mathrm{R}_{01} \gamma}{\mathrm{S}_{1}}\right) \mathrm{S}_{1}+\left(\frac{\mathrm{R}_{01}-\mathrm{R}_{0}}{\mathrm{R}_{01}-\mathrm{R}_{02}}\right)\left(\frac{\mathrm{R}_{02} \gamma}{\mathrm{S}_{2}}\right) \mathrm{S}_{2}+\gamma\right]} \\
& =\frac{1}{\left(\frac{\left(\mathrm{R}_{0}-\mathrm{R}_{02}\right) \mathrm{R}_{01}+\left(\mathrm{R}_{01}-\mathrm{R}_{0}\right) \mathrm{R}_{02}}{\mathrm{R}_{01}-\mathrm{R}_{02}}+1\right)} \\
& =\frac{1}{\left(\mathrm{R}_{0}+1\right)} .
\end{aligned}
$$

Therefore, the probability of a minor epidemic $\left(\mathrm{R}_{0} \leq 1.0\right)$ and the probability of a minor or a major epidemic $\left(R_{0}>1.0\right)$ is $R_{0} /\left(R_{0}+1\right)$. At $R_{0}=1.0$, the probabilities of either no epidemic or a minor epidemic are both 0.5 . By inference, these results apply generally to any $n$ genotype model.

When $\mathrm{R}_{0}>1.0$, the probabilities of major and minor epidemics may be determined from the basic stochastic threshold theorem, presented by Renshaw [15] and derived from extinction probabilities. In the case where an epidemic occurs and is started by a single infected animal, the probabilities of it being minor or major are $\gamma / \mathrm{N} \beta$ and $(1-\gamma / \mathrm{N} \beta)$. Using the relationship $\mathrm{R}_{0}=\mathrm{N} \beta / \gamma$, this yields probabilities of minor and major epidemics of $1 /\left(R_{0}+1\right)$ and $\left(R_{0}-1\right) /\left(R_{0}+1\right)$, respectively.

\subsection{Robustness of results to parameter estimation}

The models described above assume precise knowledge of the transmission rate. However, this is unlikely to be the case. As an example, assume that the transmission rate in a population entirely composed of the resistant genotype, is $\mathrm{R}_{02}$, and is known accurately. However, assume that $\mathrm{R}_{01}$, describing transmission in a population entirely composed of the wild-type genotype, has been underestimated by a proportion $\delta$. Therefore, $R_{01}^{*}=R_{01}+\delta R_{01}$, is the actual value of the basic reproductive ratio in the resistant population, and the true transmission in a mixed population is $\mathbf{R}_{0}^{*}=\left(\rho \mathbf{R}_{01}^{*}+(1-\rho) \mathbf{R}_{02}\right)$. However, $\rho$ will have been determined under the expectation that the basic reproductive ratio is $\mathbf{R}_{0}=\left(\rho \mathbf{R}_{01}+(1-\rho) \mathbf{R}_{02}\right)$, and the error in $\mathbf{R}_{0}^{*}$ and $\rho$ is easily calculated.

The changes in epidemic probabilities are derived similarly. Using the methodology above, the probability of no epidemic is $1 /\left(\mathrm{R}_{0}+\delta \mathrm{R}_{01} \rho+1\right)$, and the probability of a minor epidemic (or major and minor epidemic if $R_{0}^{*}>1.0$ ) is $\left(\mathbf{R}_{0}+\delta \mathbf{R}_{01} \rho\right) /\left(\mathbf{R}_{0}+\delta \mathbf{R}_{01} \rho+1\right)$. When $\mathbf{R}_{0}^{*}>1.0$, this breaks down into a probability of a minor epidemic of $1 /\left(\mathrm{R}_{0}+\delta \mathrm{R}_{01} \rho+1\right)$ and a probability of a major epidemic of $\left(\mathbf{R}_{0}+\delta \mathbf{R}_{01} \rho-1\right) /\left(\mathbf{R}_{0}+\delta \mathbf{R}_{01} \rho+1\right)$. 


\section{STOCHASTIC VERIFICATION}

\subsection{Methodology}

The formulae derived above may be verified by means of stochastic simulation, using the methodology outlined by MacKenzie and Bishop [14] for a SIR model. In brief, there are two components to a stochastic SIR epidemic model - the inter-event time and the event type. The mean time until the next event is a function of the total number of infected individuals on the farm $(\mathrm{Y})$, the total number of susceptible individuals in contact with infected animals $(\mathrm{X}), \beta$, and $\gamma$, and is given by $1 /(\mathrm{Y}(\gamma+\beta \mathrm{X}))$. The inter-event time is thus drawn from an exponential distribution as $-\ln (\mathrm{r}) /(\mathrm{Y}(\gamma+\beta \mathrm{X}))$, where $\mathrm{r}$ is a random number in $[0,1]$. This distribution yields the correct expected inter-event time as verified by calculating the expected value of $-\ln (r)$, noting that $\int \ln (\mathrm{r}) \mathrm{dr}=\mathrm{r} \ln (\mathrm{r})-\mathrm{r}$.

Given a contact rate of $c_{x y}$ between the $X$ susceptible and $Y$ infectious animals, then the probability that the event is an infection is $\beta \mathrm{YXc}_{\mathrm{xy}} / \mathrm{Y}\left(\beta \mathrm{Xc}_{\mathrm{xy}}+\gamma\right)$ and a recovery is $\gamma \mathrm{Y} / \mathrm{Y}\left(\beta \mathrm{Xc}_{\mathrm{xy}}+\gamma\right)$. This methodology can be extended to allow heterogeneity between animals, assuming there are $n$ genotypes of animals, as described in [14]. Heterogeneity in either the recovery rate $(\gamma)$ or the transmission coefficient $(\beta)$ is easily incorporated, and in this case $\gamma$ is assumed constant and $\beta_{1}$ and $\beta_{2}$ correspond to $\mathrm{R}_{01}$ and $\mathrm{R}_{02}$, respectively.

The model is implemented by introducing a single infected animal of genotype $i$, the index case. The inter-event time is calculated, the type of the first event is determined and the epidemic commences. The epidemic runs until there are no more infected animals on the farm. By recording the number of simulations that result in epidemics, the probability that an epidemic will occur and the size of epidemics can be determined. When the population is composed of mixtures of susceptible and resistant animals as described above, such that $\mathrm{R}_{0}<1.0$, all epidemics are minor epidemics, thus if the number of infected animals is greater than 1 , a minor epidemic has occurred.

To calculate $\mathrm{R}_{0}$, the output from the model was used to construct a next-generation matrix $\mathbf{M}$ describing the disease transmission rates between groups [5]. Epidemics were simulated with each genotype in turn being the index case. The element $m_{i j}$ of $\mathbf{M}$ is the number of secondary infections in genotype $j$ animals caused by the index case of genotype $i$. The $m_{i j}$ th element of the matrix obtained is divided by the number simulations performed for each index case being genotype $i . R_{0}$ is estimated as the dominant eigenvalue of M [6].

$\mathrm{R}_{0}$ and probabilities of no, minor or major epidemics were calculated for various combinations of $\mathrm{R}_{01}$ and $\mathrm{R}_{02}$. Each probability was estimated from the average of 5000 stochastic simulations in a population of 1000 animals. 


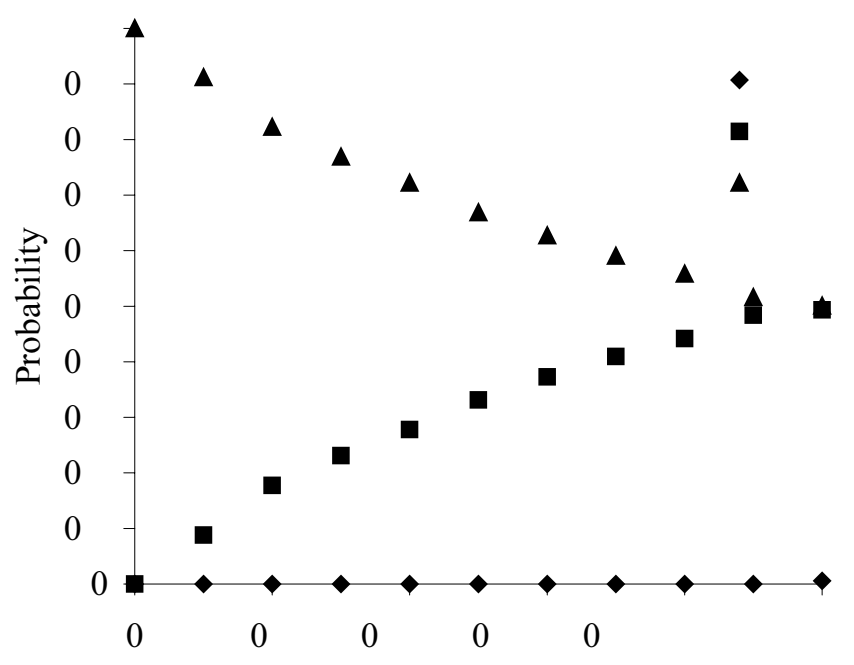

Figure 1. The simulated probability of no, minor or major epidemics in a genetically homogeneous population for expected $\mathrm{R}_{0}$ values between 0.0 and 1.0.

Observed $\mathrm{R}_{0}$ values were calculated from $\mathbf{M}$ matrices constructed from 50 replicates per simulation.

\subsection{Probabilities of epidemics}

Figure 1 shows the probability of no epidemic or minor epidemics in a single genotype population when $\mathrm{R}_{0}$ is less than 1.0. As $\mathrm{R}_{0}$ increases the probability of a minor epidemic increases and the probability of no epidemic decreases, both approaching 0.5 as $\mathrm{R}_{0}$ approaches 1.0. The points fit the expected values of $1 /\left(R_{0}+1\right)$ and $R_{0} /\left(R_{0}+1\right)$, respectively.

Figure 2 gives the probability of no epidemic, a minor or a major epidemic when the population is comprised of two genotypes. The expected basic reproductive ratio in a population composed entirely of resistant animals is (a) $\mathrm{R}_{02}=0.0$ and (b) $\mathrm{R}_{02}=0.95$. The proportions of susceptible and resistant animals are set to produce an overall expected $\mathrm{R}_{0}=1.0$. As predicted by theory, the probability that no epidemic occurs or that an epidemic is minor, dying out with a small number of infected animals, is 0.5 and the probability that an epidemic is major, infecting a large number of animals, is $0.0 . \mathrm{R}_{0}$ for all simulations is 1.0 , as expected.

\subsection{Robustness of results to parameter estimation}

To verify the theoretical results for the robustness of results to parameter estimation, populations were constructed in which $\mathrm{R}_{0}$ was underestimated. 


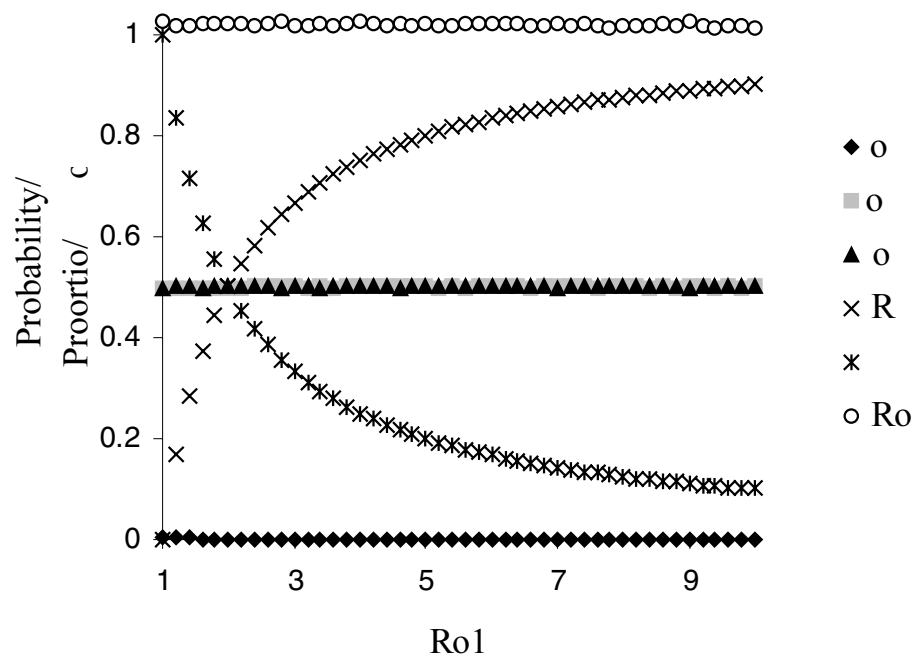

(a)

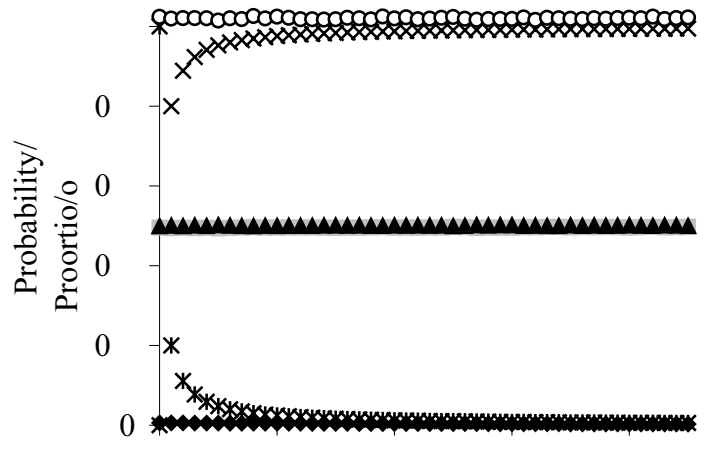

(b)

Figure 2. Required proportions of resistant animals and resulting probability of no epidemic, minor or major epidemics for a two-genotype population. Expected basic reproductive ratio in the susceptible sub-population, $\mathrm{R}_{01}$, is given on the $\mathrm{x}$-axis. Expected basic reproductive ratio for the resistant sub-population is (a) $R_{02}=0.0$, (b) $\mathrm{R}_{02}=0.95$. The observed basic reproductive ratio $\left(\mathrm{R}_{0}\right)$ for both populations is close to 1.0 in all cases.

Two approaches were investigated. In the first, the transmission rate $R_{01}$ (the wild-type genotype) was underestimated by $\delta=1.0$ and $\mathrm{R}_{02}=0.0$. In the second, it was expected that in a population composed entirely of the resistant genotype, $\mathrm{R}_{02}=0.4$, but in reality it had been underestimated by $\delta=1$.0. In both cases, simulations were conducted for $\mathrm{R}_{01}$ in the range 1.0 
to 10.0. Again, each of the probabilities was estimated from the average of 5000 stochastic simulations in a population of 1000 animals.

The results are given in Figure 3. In both cases the error in estimation had severe consequences insofar as the probability of a major epidemic rose above zero and the probability of no epidemic fall below the expected value of 0.5. The impact is particularly severe when it is $R_{01}$ (for the wildtype genotype) that is underestimated. The effect is much less severe when $R_{02}$ of the resistant genotype is underestimated, and for low $\mathrm{R}_{01}$ values this effect is trivial.

\section{DISCUSSION}

\subsection{General nature of results}

In this paper general strategies have been derived for utilising disease resistance genes, specifically genes affecting the resistance of animals to infection. As defined in this paper, resistance incorporates the transmission of infection from animals, as well as their ability to withstand infection. In particular, given knowledge of how each allele of the disease resistance gene affects the transmission of infection through a population of animals homozygous for that allele, then precise population structures can be set up to minimise the risk of epidemics. Whilst the risk and severity of epidemics will be absolutely minimised by making the population homozygous for the most resistant allele or genotype, this is not a specific requirement.

As a general summary, the population structure should be manipulated so that the overall $\mathrm{R}_{0}$ is less that 1.0. This will reduce the probability of major epidemics to zero and reduce the probability of minor epidemics below 0.5 , given a single initial infectious animal. Minor epidemics, in turn, become less likely as $\mathrm{R}_{0}$ reduces to zero. Defining appropriate population structures is mathematically straightforward, as the overall $R_{0}$ is simply the weighted average of the subpopulations.

Defining optimal population structures requires knowledge of the transmission characteristics of the pathogen, for each genotype. This is likely to be difficult information to obtain with precision. However, quantifying the impact of imprecise estimates is straightforward. The desirable outcome is that, under scenarios investigated, imprecision in the estimate of $\mathrm{R}_{02}$ (the resistant genotype) has less impact than imprecision in the estimate of $\mathrm{R}_{01}$ (the wildtype genotype).

\subsection{Application of results}

The results can be applied, in principle, to any genes that influence the resistance of animals to infections disease. However, in the case of endemic diseases or diseases where there is a consistent and heavy force of infection, the 


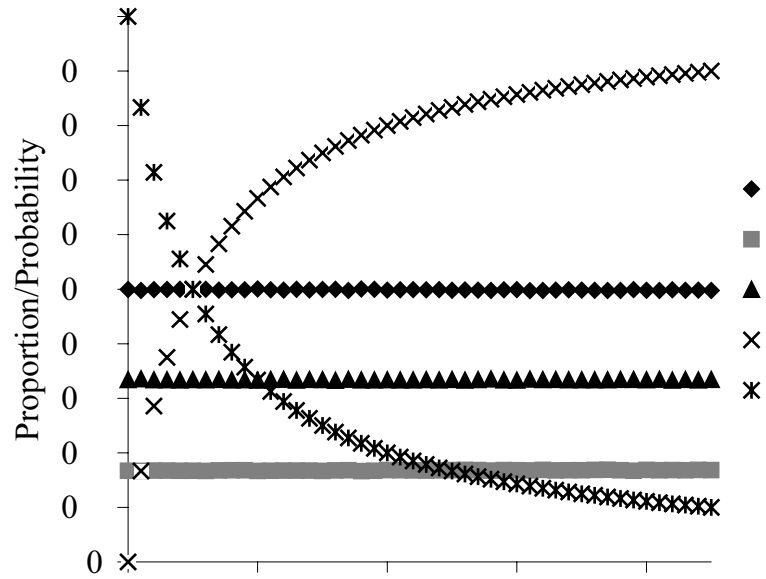

(a)

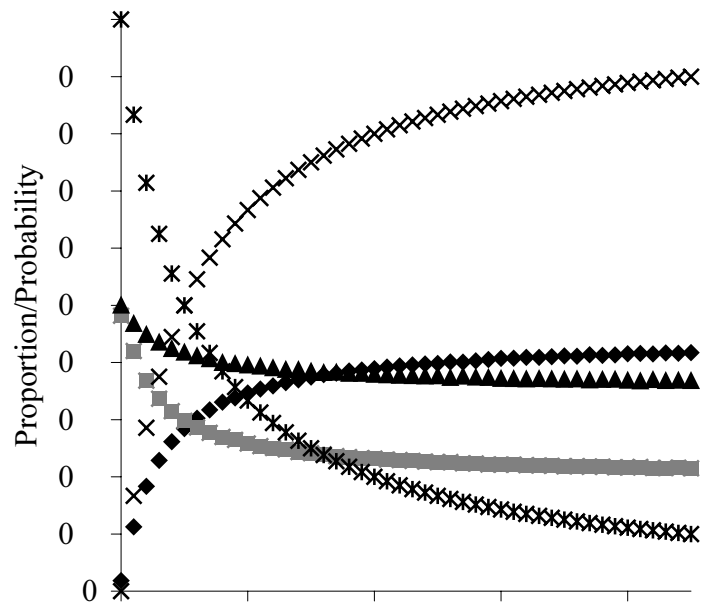

(b)

Figure 3. Consequences of underestimating transmission rate when either (a) $R_{01}$ (corresponding to the wild-type genotype) or (b) $\mathrm{R}_{02}$ (corresponding to the resistant genotype) are underestimated, in terms of the probabilities of no, minor or major epidemics. Both $\mathrm{R}_{01}$ and $\mathrm{R}_{02}$ have been underestimated by $100 \%$, and details are given in the text. 
assumption about an initial single infected animal breaks down and the epidemic probabilities will alter. When there is more than a single initial infected animal, the probability of an epidemic increases in proportion to $1-1 / \mathrm{R}_{0}^{\alpha}$, where $\alpha$ is the initial number of infected animals (derived from Renshaw [15]).

Specific disease resistance genes are still relatively rare, however major research efforts are currently underway to find such genes. Examples where genes have been found include pre-weaning and post-weaning $E$. coli diarrhoea in pigs [8], PrP alleles conferring scrapie resistance [4] and MHC haplotypes conferring enhanced resistance to Marek's disease. The results presented here apply to each of these diseases. Selection for scrapie resistance has been previously investigated using a model combining host genetics and disease epidemiology [17], with the prediction that scrapie should be eradicated before the population is completely genetically resistant. This agrees with the theory presented here.

Confidence in finding of disease resistance genes may be gained from the fact that when specific diseases are subjected to rigorous scrutiny, genetic variation in host resistance is invariably seen. For example, there is documented genetic variation in host resistance or tolerance for over 50 domestic livestock diseases [3]. Even when there are no diseases of overriding importance, genetic variation in liability to infection by certain classes of pathogen can still be demonstrated [12]. Additionally, considerable genetic variation in immune response has been demonstrated in a number of studies $[10,9,16]$. Hitherto, strategies for utilising genes contributing to this variation were absent. This paper provides a framework for developing these strategies.

\subsection{Pathogen evolution considerations}

Pathogen evolution potentially negates the attempts to use genetics to control an infectious disease and it impacts upon genetic management strategies. Two principles are important: (i) absolute risks of pathogen evolution are inestimable, only relative risks for different genetic management strategies may be calculated; (ii) in general terms mutations make organisms less fit, unless there is a niche in which the mutation is beneficial.

Consider a population of hosts selected for resistance and a supra-population of wildtype hosts that forms the reservoir for the pathogens. There will not be strong selection pressure for pathogen evolution in the managed population if $\mathrm{R}_{0}$ is greater than 1.0, nor is there strong selection pressure in the suprapopulation. More generally, selection pressures that do exist will be a trade-off between pathogen transmission and virulence, i.e. host mortality induced by infection [11]. However, genetic management strategies reducing $\mathrm{R}_{0}$ below 1.0 potentially create selective advantages for pathogens carrying specific mutations. Of importance are both the mechanism and genetic heterogeneity of host resistance, as both will influence relative risks. 
Genetic resistance has been formulated in terms of the transmission rate, $\beta$, which is the product of susceptibility (probability of becoming infected) and infectivity (transmission of infection to other individuals). If resistance is due to a reduction in susceptibility and genetic management eliminates all epidemics, then the pathogen cannot establish itself in the host population and evolution risks are minimal. However, minor epidemics create a niche for pathogens with specific mutations as the population maintains the pathogen for a short period of time and evolution potentially gives the pathogen the ability to further invade the population. Therefore, if resistance is due to reduced susceptibility, genetic management strategies that reduce the probability of minor epidemics will also reduce the probability of pathogen evolution. If resistance is due to reduced infectivity, the dynamics alter. In this case, an infected animal may already exist in the population, but this animal is unable to transmit infection to other animals. Thus, the niche for evolution exists whether or not there is a minor epidemic, however minor epidemics increase the opportunity for evolution. The differing implications of reducing the probability of infection or reducing subsequent transmission of infection have previously been illustrated in the context of vaccine control by Gandon et al. [11]. Please note that the arguments used here are for mutations affecting pathogen transmission; much of the (co)evolutionary theory deals with long-term trade-offs between pathogen transmission and virulence.

Genetic heterogeneity in resistance can be of two types: genetic differences between different groups of animals or individual animals having resistance alleles at more than one locus. In the case where different groups of animals are resistant by different mechanisms, the arguments are the same as above, except that the niche of newly susceptible animals is now much narrower when the pathogen mutates successfully. The $n$ genotype model is relevant and, for the ith group, $\mathrm{R}_{0 \mathrm{i}}$ is now larger than it previously was. Depending upon the mix of genotypes, this may not have a substantial effect on overall disease transmission dynamics: the greater the heterogeneity the less the overall effect. Within-animal heterogeneity, i.e. two or more resistance mechanisms per animal, will pose greater evolutionary challenges to the pathogen, as two independent mutations will be required to overcome the hosts defence mechanisms. Moreover, these mutations must occur in the same pathogen and occur close together in time to overcome possible selective disadvantages of mutations with no immediate benefit. Therefore, within-animal heterogeneity in resistance is likely to reduce the risk of pathogen evolution.

\subsection{Concluding remarks}

Methodologies now exist for breeders to make appropriate use of disease resistance genes, and in general terms it will not be necessary to fix the favourable alleles within a population. However, application of these principles 
requires the breeder to understand the disease, the mechanisms of the gene and the nature of the infectious challenge faced by animals. For example, these strategies will not be appropriate if the gene confers tolerance of infection. Furthermore, these strategies will not be appropriate if the force of infection results from a continuous challenge from an essentially-infinite reservoir of infection. Such situations have previously been considered by van der Waaij et al. $[18,19]$.

Breeders should also be aware that reliance upon a single gene (or allele) increases the risk of pathogens evolving to overcome the genetic strategy. However, this is no different from any other disease control strategy that relies upon a single mechanism. Risks will be reduced by combining strategies and utilising, where possible, more than one resistance gene.

\section{ACKNOWLEDGEMENTS}

We wish to thank BBSRC for funding.

\section{REFERENCES}

[1] Anderson R.M., May R.M., Population biology of infections diseases: Part 1, Nature 280 (1979) 361-329.

[2] Anderson R.M., May R.M., Infectious diseases of humans. Dynamics and control, Oxford University Press, Oxford, 1992.

[3] Bishop S.C., Chesnais J., Stear M.J., Breeding for disease resistance: issues and opportunities, in: Proceedings of the 7th World Congress on Genetics Applied to Livestock Production, Montpellier, INRA, Castanet-Tolosan, France, 18-24 August 2002, CD-Rom communication 13-01.

[4] Dawson M., Hoinville L.J., Hosie B.D., Hunter N., Guidance on the use of PrP genotyping as an aid to the control of clinical scrapie, Vet. Record 142 (1998) 623-625.

[5] De Jong M.C.M, Diekmann O., Heesterbeek J.A.P., The computation of $\mathrm{R}_{0}$ for discrete-time epidemic models with dynamic heterogeneity, Math. Biosci. 119 (1994) 97-114.

[6] Diekmann O., Heesterbeek J.A.P., Metz J.A.J., On the definition and the computation of the basic reproduction ratio $\mathrm{R}_{0}$ in models for infectious diseases in heterogeneous populations, J. Math. Biol. 28 (1990) 365-382.

[7] Dushoff J., Levin S., The effects of population heterogeneity on disease invasion, Math. Biosci. 128 (1995) 25-40.

[8] Edfors-Lilja I., Wallgren P., Escherichia coli and Salmonella diarrhoea in: Axford R.F.E., Bishop S.C., Nicholas F.W., Owen J.B. (Eds), Breeding for Disease Resistance in Farm Animals, 2nd edn., CABI Publishing, Wallingford, Oxon, UK, 2000, pp. 253-267. 
[9] Edfors-Lilja I., Wattrang E., Marklund L., Moller M., Andersson-Eklund L., Andersson L., Fossum C., Mapping quantitative trait loci for immune capacity in the pig, J. Immunol. 161 (1998) 829-835.

[10] Edfors-Lilja I., Wattrang E., Andersson L., Fossum C., Mapping quantitative trait loci for stress induced alterations in porcine leukocyte numbers and functions, Anim. Genet. 31 (2000) 186-193.

[11] Gandon S., MacKinnon M.J., Nee S., Read A.F., Imperfect vaccines and the evolution of virulence, Nature 414 (2001) 751-756.

[12] Henryon M., Berg P., Jensen J., Sorensen S., Genetic variation for resistance to clinical and subclinical diseases exists in growing pigs, Anim. Sci. 73 (2001) 375-387.

[13] MacKenzie K.M., Bishop S.C., A discrete-time epidemiological model to quantify selection for disease resistance, Anim. Sci. 69 (1999) 543-551.

[14] MacKenzie K.M., Bishop S.C., Developing stochastic epidemiological models to quantify the dynamics of infectious diseases in domestic livestock, J. Anim. Sci. 79 (2001) 2047-2056.

[15] Renshaw E., Modelling Biological Populations in Space and Time, Cambridge University Press, Cambridge, 1991.

[16] Strain S., Bishop S.C., Henderson N., Holmes P.H., McKellar Q.A., Mitchell S., Stear M.J., The genetic control of IgA activity and its association with parasite resistance in naturally infected sheep, Parasitology 124 (2002) 545-552.

[17] Stringer S.M., Hunter N., Woolhouse M.E.J., A mathematical model of the dynamics of scrapie in a sheep flock, Math. Biosci. 153 (1998) 79-98.

[18] Van der Waaij E.H., Pijma P., Bishop S.C., van Arendonk J.A.M., Modeling selection for production traits under constant infection pressure, J. Anim. Sci. 78 (2000) 2809-2820.

[19] van der Waaij E.H., Bijma P., Bishop S.C., van Arendonk J.A.M., Utilising genetic markers for disease resistance to improve production under constant infection pressure, J. Anim. Sci. 80 (2002) 322-329.

To access this journal online: www.edpsciences.org 